Plateforme numérique d'apprentissage pour développer les compétences de compréhension orale en FLE

\title{
Plateforme numérique d'apprentissage pour développer les compétences de compréhension orale en FLE
}

\author{
Dr. Walid Sobhi AL ADL \\ Maître de conférences de curricula et de méthodologies \\ Faculté de Pédagogie, Université de Damiette \\ walidaladl@yahoo.com
}


Plateforme numérique d'apprentissage pour développer les compétences de compréhension orale en FLE

\section{منصة تعلم رقمية لتتمية مهارات القهم الثظفوي في اللغة القرنسية كلغة أجنبية \\ د. وليد صبحى عبد الحميد العدل \\ مدرس المناهج وطرق التدريس - كلية التربية- جامعة دمياط \\ walidaladl@yahoo.com}

مستخلص:

يهدف البحث الحالي إلى التحقق من أثز منصة تعلم رقمية على تتمية مهارات

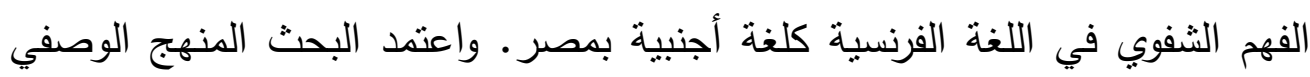
التحليلي لعرض المفاهيم والأدبيات المتعلقة بمهارات الفهم الثفوي، وتوظيف منصات التعلم الرقمية في تتمية مهارات الفهم الثفوي للغة الفرنسية. كما اعتمد المنهج شبه التجريبي بتطبيق أدوات البحث للتحقق من أثرها على تتمية مهارات الفهم الثفوي لدى Quiz vidéo ( عينة البحث المستهدفة. وتمتلت أدوات البحث في منصة تعلم رقمية de FLE (قبلي/بعدي) وفقاً للمستوبين الأول والثاني من الإطار الأوروبي المرجعي للغات (CECR) بكلية التربية، جامعة دمياط، كمجموعة تجريبية. وقد أسفرت نتائج البحث عن وجود

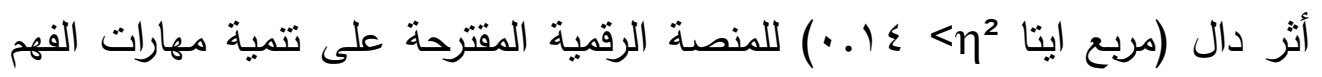
الثفوي في اللغة الفرنسية كلغة أجنبية لدى عينة البحث.

الكلمات المفتاحية: منصة تعلم رقمية - مهارات الفهم الثفوي- الإطار الأوروبي المرجعي. 
Plateforme numérique d'apprentissage pour développer les compétences de compréhension orale en FLE Dr . Walid Sobhi AL ADL

\title{
Plateforme numérique d'apprentissage pour développer les compétences de compréhension orale en FLE
}

\author{
Dr. Walid Sobhi AL ADL \\ Maître de conférences de curricula et de méthodologies \\ Faculté de Pédagogie, Université de Damiette \\ walidaladl@yahoo.com
}

\section{Résumé}

La recherche actuelle a pour but de vérifier l'effet d'une plateforme numérique d'apprentissage sur le développement de la compétence de compréhension orale en FLE. Nous avons utilisé la méthode descriptive et analytique en exposant les concepts, les ouvrages et les études antérieurs portant sur la compétence de la compréhension orale et les plateformes numériques d'apprentissage au service de la compréhension orale. Nous avons aussi utilisé la méthode quasi-expérimentale en appliquant les outils de la recherche pour vérifier leurs impacts sur le développement de la compétence de compréhension orale chez l'échantillon ciblé. Nous avons pour cela conçu une plateforme numérique d'apprentissage (Quiz vidéo de FLE), une série d'activités de compréhension orale déjà préparée et un pré/posttest pour les niveaux A1 et A2 du Cadre Européen Commun de Référence pour les langues (CECR, 2001). Notre échantillon se compose de 30 étudiant(e)s de deuxième année, section de français, à la faculté de pédagogie, université de Damiette comme groupe expérimental. Les résultats de cette recherche permettent de dire que la plateforme numérique proposée a eu un impact significatif $\left(\eta^{2}>0.14\right)$ sur le développement de la compétence de compréhension orale en FLE pour nos étudiants de niveau débutant.

Most clés : Plateforme numérique - compréhension orale - CECR 
Plateforme numérique d'apprentissage pour développer les compétences de compréhension orale en FLE

Dr. Walid Sobhi AL ADL

\section{Introduction}

L'enseignement/apprentissage de la langue française, comme deuxième langue étrangère, vise à développer les compétences orales et écrites de l'apprenant. Étant donné qu'acquérir une langue étrangère c'est acquérir les habitudes de communication propres à une communauté. La priorité doit être donnée à l'aspect oral de la langue parce que celle-ci est parlée avant d'être écrite. En outre, il faut s'intéresser aux compétences orales parce qu'elles se lient avec les autres compétences écrites (Mohammed, 2007). La compétence de compréhension orale joue un rôle primordial dans l'apprentissage de la langue. C'est par l'oreille que l'homme prend le premier contact avec le monde. La compréhension orale est la compétence de la langue la plus fréquemment utilisée en communication humaine. Selon les recherches antérieures, le temps que l'on passe à écouter est deux fois plus important que celui que l'on passe à parler, le quadruple du temps passé à lire et le quintuple du temps passé à écrire (Hyslop \& Tone, 1989). D'où apparaissent l'importance et la priorité de la compréhension orale dans l'apprentissage d'une langue étrangère.

Depuis le début des années 2000, avec le développement rapide des Technologies de l'Information et de la Communication (TIC), les didacticiens mettent l'accent sur l'utilisation des médias numériques, et plus récemment des plateformes d'apprentissage en ligne, afin de motiver les apprenants et rendre l'enseignement/apprentissage plus efficace. L'utilisation des nouvelles technologies pourrait inciter les apprenants à apprendre et améliorer leurs compétences langagières. Selon Spiezia (2001), l'introduction des nouvelles technologies dans l'enseignement d'une langue étrangère permet de mieux organiser les parcours d'apprentissage individualisés et d'envisager un apprentissage en

\footnotetext{
*Walid AL ADL, Maître de conférences à la Faculté de Pédagogie de l’Université de Damiette
} 
Plateforme numérique d'apprentissage pour développer les compétences de compréhension orale en FLE Dr . Walid Sobhi AL ADL

autonomie. Ces nouvelles technologies numériques offriraient ses activités d'apprentissage plus attrayantes pour l'apprenant, et pourraient favoriser, dans le cadre d'une approche intégrée, l'apprentissage d'une langue étrangère à travers la pluralité des ressources utilisées. De là naît la nécessité d'analyser l'impact des nouvelles technologies qui peuvent faciliter ou au moins améliorer l'enseignement/apprentissage de la langue étrangère. Notre recherche vise à vérifier l'effet d'une plateforme numérique d'apprentissage sur le développement de la compétence de compréhension orale chez les étudiants de la section de français à la faculté de pédagogie de Damiette.

\section{Problématique de la recherche}

De nombreuses études ont montré que le niveau des étudiants égyptiens dans les facultés de pédagogie en français langue étrangère est assez faible surtout à l'oral (Al Adl, 2017 ; El-Alawy, 2015 ; Omar, 2014 ; Mohammed, 2012 ; Mohamed, 2011 ; El-Fakharany, 2010 ; Hafez, 2009). Ces études ont également recommandé de développer les compétences orales des apprenants à travers l'utilisation des nouveaux médias. Avec l'emploi des outils des TIC, et plus précisément des plateformes numériques d'apprentissage, il est possible de créer un environnement numérique d'apprentissage interactif grâce au multimédia qui regroupe la voix, l'image fixe, la vidéo, l'animation et les textes. Il est également possible de présenter des tâches communicatives simulées ou authentiques afin que l'apprenant puisse concevoir et s'entraîner à une communication proche de la réalité (Salem, 2002 ; Huver et Springer, 2011).

Notre recherche, qui s'intéresse à des étudiants de niveau débutant (A1 et A2), essaie de répondre à la question principale suivante :

Quelle est l'effet d'une plateforme numérique d'apprentissage sur le développement de la compétence de compréhension orale en FLE? 
Plateforme numérique d'apprentissage pour développer les compétences de compréhension orale en FLE Dr . Walid Sobhi AL ADL

Issues de la question principale, d'autres sous-question :

1) Quel est le niveau réel des étudiants de l'échantillon ciblé à la compréhension orale?

2) Comment améliorer la compréhension orale chez l'échantillon de la recherche ?

3) Quel est l'effet de la plateforme proposée sur la compréhension orale de l'échantillon ciblé ?

\section{Objectifs de la recherche}

La recherche actuelle a pour but de :

1- identifier la compétence de compréhension orale chez les futurs-enseignants des facultés de pédagogie de Damiette ;

2- développer les aptitudes de la compréhension orale nécessaires pour les étudiants de la section de français à la faculté de pédagogie de Damiette.

3- adapter une plateforme numérique d'apprentissage pour développer quelques aptitudes en compréhension orale chez l'échantillon de la recherche ;

4- vérifier l'effet de la plateforme proposée sur le développement de la compréhension orale chez les étudiants de l'échantillon.

\section{Outils de la recherche}

Afin d'obtenir des données pertinentes permettant de répondre aux questions précédentes, nous avons élaboré :

1- Un test de la compétence de compréhension orale pour les niveaux A1 et A2 du CECR ;

2- Une plateforme numérique d'apprentissage pour développer la compétence de compréhension orale pour les niveaux A1 et A2 du CECR en FLE chez l'échantillon de la recherche.

\section{Limites de la recherche}

La recherche actuelle s'est limitée à : 
Plateforme numérique d'apprentissage pour développer les compétences de compréhension orale en FLE Dr . Walid Sobhi AL ADL

a) Un échantillon ciblé d'étudiants de deuxième année, section de français à la faculté de pédagogie de Damiette, comme groupe expérimental ;

b) La compétence de compréhension orale pour les niveaux A1 et A2 du CECR et les ressources multimédias appropriées à ces niveaux ;

c) Le deuxième semestre de l'année 2017/2018 pour expérimenter la plateforme proposée sur l'échantillon de la recherche.

\section{Hypothèses de la recherche}

Cette recherche a essayé d'examiner les hypothèses suivantes :

1) Il existe une différence significative au niveau $\leq 0.05$ entre les moyennes des notes des étudiants au pré/posttest de compréhension orale en faveur du posttest.

2) La plateforme numérique proposée a une taille d'effet $\eta^{2} \geq$ 0.14 sur le développement de la compétence de compréhension orale en FLE pour l'échantillon de la recherche.

\section{Cadre théorique}

\section{La compétence de compréhension orale}

Cette recherche met l'accent sur le développement de la compétence de compréhension orale. Nous sommes persuadés qu'un bon apprentissage d'une langue étrangère passe en premier temps par une maîtrise de la compréhension orale. Il est reconnu que les enfants acquièrent, dans une langue maternelle, l'aptitude à écouter et par la suite à comprendre bien avant d'apprendre à parler, à écrire et à lire. Pareillement, les apprenants d'une langue étrangère ont besoin d'une bonne maîtrise de la compréhension orale dès le début de leur apprentissage. Ce besoin est encore plus grand dans un pays comme l'Égypte où l'anglais domine totalement et où les étudiants entendent très peu de français hors des cours. La compréhension orale est une compétence primordiale dans l'apprentissage des langues étrangères parce qu'il favorise la conscience phonologique 
Plateforme numérique d'apprentissage pour développer les compétences de compréhension orale en FLE

Dr . Walid Sobhi AL ADL

et syntaxique, l'acquisition du vocabulaire et des structures langagières, les progrès en lecture et en écriture et réalise en fin l'interaction entre l'homme et le monde où il vit. Il va de soi, d'une part, que l'apprenant ne peut pas parler une langue sans l'écouter ; et d'autre part, s'il possède l'habileté de la compréhension orale, il devient évidemment plus capable de parler, de lire et d'écrire et donc d'interagir. La compréhension orale «constitue ainsi le socle de l'apprentissage d'autres compétences langagières à communiquer (parler, lecture, écriture interaction et médiation) » (Al Adl, 2017). Par ailleurs, l'écoute permet aux apprenants de se familiariser avec les sons de la langue, qu'elle soit maternelle, seconde ou étrangère et les amène à «s'appuyer sur les éléments du texte et les connaissances personnelles pour comprendre un message oral. » (Ferroukhi, 2009 : 273). D'où apparaissent l'importance et la priorité de la compréhension orale pour le français langue étrangère.

Cependant, la compréhension orale demeure, comme le rappelle Dankova (2013: 165), «une des difficultés majeures des apprenants familiarisés davantage avec la langue écrite qu'avec la langue orale ». La faiblesse de la compréhension orale les empêche d'accéder au contenu écouté sans l'aide d'un enseignant prêt à les guider pendant l'écoute; c'est pour cela, les apprenants perdent rapidement leur motivation à compléter l'écoute d'un document sonore et se trouvent mal préparés pour comprendre et échanger en français avec des natifs.

La compréhension orale vise en premier lieu à faire acquérir graduellement à l'apprenant des stratégies d'écoute et en deuxième lieu de compréhension d'énoncés à l'oral. Elle implique donc la combinaison de deux processus: l'écoute des sons émis par l'émetteur et la compréhension du sens des mots prononcés, le message du locuteur (Leroy, 2016 :5).

Les auteurs du CECR spécifient que l'utilisateur de la langue comme auditeur reçoit et traite, dans une activité de réception orale, un message parlé produit par un ou plusieurs locuteurs. Dans ce contexte, le CECR insiste sur l'importance de l'opération de compréhension. Il distingue trois types d'activités 
Plateforme numérique d'apprentissage pour développer les compétences de compréhension orale en FLE

Dr. Walid Sobhi AL ADL

de compréhension: une compréhension globale (elle vise à découvrir la signification générale du document), une compréhension sélective (elle vise à repère certaines informations cherchées par l'auditeur) et une compréhension détaillée (elle consiste à reconstituer entièrement le document) (Conseil de l'Europe, $2001: 54,126)$.

Afin de mieux maitriser l'écoute et de mieux comprendre l'oral, il faut prendre en considération les facteurs qui peuvent, dans certaines conditions, développer la compréhension en langue étrangère, tels que la réécoute du document sonore (Elkhafaifi, 2005), la régulation de l'écoute grâce à l'outil numérique (Roussel et al., 2008), ou encore l'anticipation par l'activation des connaissances préalables de l'apprenant sur le document à écouter (Vandergrift et Goh, 2012). Le développement de cette compétence réside dans "l'interaction des savoirs et des savoirfaire requis. »En outres, la bonne compréhension exige «la reconnaissance de la signification d'un discours ou d'une phrase et l'identification de leur fonction communicative (fonction référentielle, expressive, conative, phatique, métalinguistique et poétique» (Alrabadi, 2011: 22). Au total, «la préparation à l'écoute et sa mise en contexte permettent aux auditeurs de mieux construire le sens » (Roussel et Tricot, $2015: 2$ ).

\section{La plateforme numérique d'apprentissage et la compréhension orale}

L'utilisation des plateformes numériques devient de plus en plus très répandue dans l'enseignement supérieur en raison des potentialités souples qu'elles offrent dans les différentes disciplines des sciences sociales s'intéressant au numérique. Cette utilisation s'inscrit dans un environnement d'apprentissage interactif enrichi par le multimédia, qui regroupe la voix, l'image fixe, la vidéo, l'animation et les textes. Une plateforme numérique d'apprentissage multimédia peut présenter aujourd'hui des situations communicatives simulées ou authentiques de la vie réelle et permet ainsi d'entrainer l'apprenant à entrer en contact 
Plateforme numérique d'apprentissage pour développer les compétences de compréhension orale en FLE Dr . Walid Sobhi AL ADL

avec les natifs francophones. Ce type de numérisation est basé sur le principe d'individualisation de l'enseignement des langues. L'apprenant peut apprendre seul à son propre rythme, ou en petits groupes et obtenir immédiatement la rétroaction corrective.

L'office québécois de la langue française (2005) a défini ces plateformes comme "système informatique destiné à automatiser les diverses fonctions relatives à l'organisation des cours, à la gestion de leur contenu, au suivi des progrès des participants et à la supervision des personnes responsables des différentes formations (...). Elles permettent aussi «de gérer et de donner accès à un ensemble d'activités et de ressources pédagogiques » (George et Derycke, 2005 : 52).

De nombreuses plateformes de formation en ligne offrent des activités basées sur des extraits de films, d'un journal télévisé ou d'autres documents vidéos, comme par exemple le site de TV5 Monde (Dankova, 2013). Ces activités numériques permettent aux apprenants d'y accéder gratuitement et de réécouter leur contenu à n'importe quel moment et à n'importe quel lieu.

Dans cette recherche, nous proposons l'utilisation de la plateforme des Quiz vidéo de FLE (http://www.flevideo.com/index.php) comme l'une des plateformes numériques qu'on peut utiliser dans le développement de la compréhension orale. Cette plateforme permet aux enseignants de créer leurs propres activités vidéos avec des quiz de FLE et de les gérer aisément. De plus, il présente de nombreuses activités appropriées aux apprenants de différents niveaux (A1 - B2 selon les normes du CECR) avec des quiz vidéo de FLE amusants et gratuits. L'utilisation de cette plateforme ne requiert pas de connaissances poussées en informatique. En outre, elle est gratuite et utilisable aussi bien sur un PC que sur un Mac. Les activités créées avec cette plateforme seront accessibles à n'importe quel lieu et à n'importe quel moment, autrement dit aux apprenants guidés par l'enseignant à les consulter. De surcroît, cette plateforme permet aux enseignants de créer leur code de 
Plateforme numérique d'apprentissage pour développer les compétences de compréhension orale en FLE Dr . Walid Sobhi AL ADL

professeur. L'apprenant consulte en ligne les contenus pédagogiques qui lui sont recommandés. Il doit pouvoir organiser son travail et avoir une vue de son évolution. Il effectue des exercices, s'autoévalue et peut envoyer à son enseignent les scores de ses quiz en saisissant son nom et le code du professeur dans le formulaire des résultats du quiz. Tous ces avantages rendent cette plateforme un terreau fertile et un environnement favorable pour créer et gérer des activités d'entraînement en vue de développer la compétence de compréhension orale.

http://www.flevideo.com/fle_video_quiz_beginning.php?id=7321

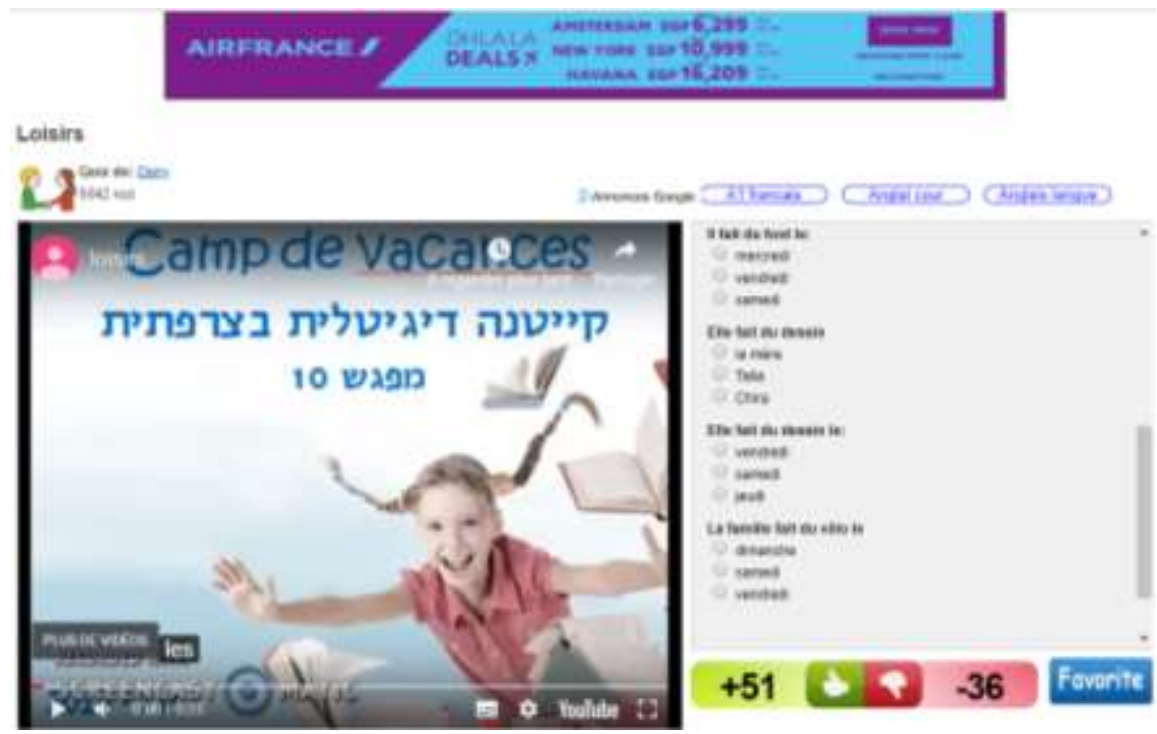

Figure 1 : Écran de la page d'activités numériques de compréhension orale

La plateforme sert à concevoir des activités de compréhension orale à partir des vidéos disponibles sur YouTube ou de propres vidéos téléchargés et sauvegardés sur le bureau de l'ordinateur personnel. La vidéo choisie sera associée à la questionnaire désirée et visionnée partiellement ou intégralement sans quitter le questionnaire. Il est conseillé de conserver sa propre copie de la vidéo utilisée au cas où elle serait retirée de YouTube. 
Plateforme numérique d'apprentissage pour développer les compétences de compréhension orale en FLE

Dr . Walid Sobhi AL ADL

Cette plateforme se caractérise par la possibilité d'ajouter et de modifier des questions, des transcriptions ou des commentaires sans altérer la vidéo. Les apprenants peuvent revenir sur un passage du document vidéo et le réécouter pour répondre aux questions sur son contenu. De plus, les apprenants peuvent afficher, en cas de difficulté, les sous-titres du contenu sonore pour les aider à comprendre ce qu'ils trouvent difficile à comprendre; au besoin, on peut ajouter des transcriptions des passages jugés trop difficiles ou encore des remarques particulières. Les quiz sont intégrés à côté de la vidéo sous forme des questions à choix-multiples dont le professeur détermine les choix corrects. Les apprenants peuvent, grâce à la correction automatique, afficher leurs résultats, mesurer leurs progrès tout au long de l'apprentissage et vérifier leurs acquis en fin de parcours. En outre, les apprenants peuvent envoyer automatiquement leurs résultats à leur formateur en utilisant le Code $d u$ professeur déjà préparé.

La plateforme Quiz vidéo de FLE permet de développer des tests formatifs ou des examens en ligne. L'option d'examen propose une évaluation automatique du travail de l'apprenant. L'enseignant détermine, lors de la conception de l'examen, la réponse correcte à chacune des questions, et le logiciel calcule le résultat et affiche les bonnes réponses. En d'autres termes, la facilité d'utilisation et les fonctionnalités de cette plateforme permettent de concevoir rapidement des activités de compréhension orale basées sur des documents audiovisuels authentiques choisis par l'enseignant.

À chaque étape de la conception du questionnaire, le site propose de l'aide en expliquant la démarche à suivre. L'enseignant modifie au besoin les questions sauvegardées. De plus, la plateforme prévoit un espace pour ajouter des commentaires des apprenants, de l'enseignant ou d'autres visiteurs, ce qui permet d'échanger les opinions entre eux. 
Plateforme numérique d'apprentissage pour développer les compétences de compréhension orale en FLE Dr . Walid Sobhi AL ADL

\section{Méthodologie de la recherche}

\section{Méthodes de la recherche}

Nous avons utilisé dans cette recherche une méthode de type quasi-expérimental en appliquant les outils de la recherche pour vérifier leurs impacts sur le développement de la compétence de compréhension orale chez l'échantillon de la recherche ciblé. En outre, nous avons recouru à la méthode descriptive pour en développant le cadre théorique de la recherche.

\section{2. Échantillon de la recherche}

Nous avons choisi trente étudiants de deuxième année, section de français, à la faculté de pédagogie de Damiette, de l'année universitaire 2017/2018, comme groupe expérimental. Nous avons choisi ces étudiants parce qu'ils s'entraînent durant la deuxième année aux compétences professionnelles et communicatives dans le cours du micro-enseignement. Ils sont sur le point de démarrer l'enseignement du français langue étrangère.

\section{Expérimentation des outils de la recherche}

Nous avons appliqué un pré-test à l'échantillon de la recherche le 12 février 2018. Ce pré-test a pour objectif premier d'évaluer la compétence de compréhension orale des étudiants au début du parcours. Les résultats du pré-test ont indiqué la faiblesse du niveau des apprenants en ce qui concerne la compréhension orale en français.

L'expérimentation de la plateforme proposée a eu lieu du 17 février 2018 au 12 avril 2018, durant le deuxième semestre de l'année universitaire 2017/2018, à travers les activités numériques déjà préparées. Elle a duré deux mois au rythme d'une activité par semaine pour bien s'entraîner à la compréhension de l'oral, une semaine au début pour les notions préliminaires et une autre à la fin pour les étudiants retardataires. 
Plateforme numérique d'apprentissage pour développer les compétences de compréhension orale en FLE Dr . Walid Sobhi AL ADL

Nous avons appliqué un posttest à l'échantillon de la recherche le 14 avril 2018. Ce posttest a pour objectif de vérifier le degré de progrès réalisé après avoir s'entrainé aux activités proposées et l'effet de la plateforme numérique sur le développement de la compréhension orale chez les participants. Nous avons ainsi fini notre expérience, qui a duré deux mois et deux jours, du 12 février 2018 (pré-évaluation) au 14 avril 2018 (post-évaluation).

\section{Résultats et conclusion}

Nous présentons les résultats obtenus selon les hypothèses de la recherche :

Pour vérifier la validité de la première hypothèse, qui stipule qu' " il existe une différence significative au niveau $\leq 0.05$ entre les moyennes des notes des étudiants au pré/posttest de compréhension orale en faveur du posttest », nous avons calculé d'abord le pourcentage, la moyenne arithmétique des notes des étudiants au pré/posttest de compréhension orale en utilisant le tableur Excel (annexe 1). Ensuite, nous avons appliqué le test T pour échantillons appariés afin de comparer les moyennes des résultats obtenus par les participants au pré/posttest de compréhension orale. Nous avons obtenu les résultats suivants (Tableau 1) :

Tableau 1: Pourcentages, moyennes et valeur de $T$ au pré/posttest de compréhension orale

\begin{tabular}{|c|c|c|c|c|c|c|}
\hline Test & $\mathbf{N}^{*}$ & $\mathbf{P}^{\dagger}$ & $\mathbf{M}^{\ddagger}$ & $\mathbf{E . T}^{\S}$ & $\mathbf{T}^{* *}$ & $\mathbf{S}^{\dagger \dagger}$ \\
\cline { 1 - 7 } Posttest de compréhension orale & 30 & $62 \%$ & 15.57 & 2.501 & \multirow{2}{*}{16.84} & \multirow{2}{*}{0.000} \\
\cline { 1 - 6 } Pré-test de compréhension orale & 30 & $33 \%$ & 8.30 & 2.152 & 7 & \\
\hline
\end{tabular}

*Nombre des apprenants

${ }^{\dagger}$ Pourcentage

${ }^{\$}$ Moyenne arithmétique

$\S$ Écart-type

${ }^{* * *}$ Valeur du test T

${ }^{\dagger}$ Signification 
Plateforme numérique d'apprentissage pour développer les compétences de compréhension orale en FLE Dr . Walid Sobhi AL ADL

Le tableau 1 ci-dessus montre que le pourcentage de réponses des apprenants au posttest est de $62 \%$ alors qu'il était de $33 \%$ au pré-test. La moyenne au posttest est de 15.57 alors qu'elle était de 8.30 au pré-test. Par ailleurs, la valeur du test T est de 16.847 au niveau de signification (0.000). Cela signifie qu'il existe une différence significative au niveau $<0.05$ entre les moyennes des notes des étudiants au pré/posttest de compréhension orale en faveur du posttest. Donc, notre première hypothèse est confirmée.

Pour vérifier la validité de la deuxième hypothèse, qui stipule qu' «la plateforme numérique proposée a une taille d'effet $\eta^{2} \geq 0.14$ sur le développement de la compétence de compréhension orale en FLE pour 1'échantillon de la recherche », nous avons calculé l'eta-carré $\left(\eta^{2}\right)$ qui indique la taille d'effet (effect size) avec l'équation et le tableau 2 suivants :

$\eta^{2}=\frac{T^{2}}{\left(T^{2}+N-1\right)}$

$\eta^{2}:$ Eta-carré

$\mathrm{T}^{2}$ : Carré du test $\mathrm{T}$

$\mathrm{N}$ : Nombre des apprenants

$\mathrm{N}-1$ = Degré de liberté (D.L)

$\eta^{2}=\frac{283.8}{283.8+29}=0.907$ 
Plateforme numérique d'apprentissage pour développer les compétences de compréhension orale en FLE Dr . Walid Sobhi AL ADL

\section{Tableau 2: Valeur de l'eta-carré}

\begin{tabular}{|c|c|c|c|c|c|}
\hline $\begin{array}{c}\text { Variable } \\
\text { indépendante }\end{array}$ & $\begin{array}{c}\text { Variable } \\
\text { dépendante }\end{array}$ & $(\mathbf{T})^{*}$ & $\left(\mathbf{T}^{2}\right)^{\dagger}$ & $\begin{array}{c}(\mathbf{D} . \mathbf{L})^{\ddagger} \\
\ddagger\end{array}$ & ${\mathbf{( \eta ^ { 2 }}}^{\S}$ \\
\hline $\begin{array}{c}\text { La plateforme } \\
\text { proposée }\end{array}$ & $\begin{array}{c}\text { La compétence de } \\
\text { compréhension orale }\end{array}$ & $\begin{array}{c}16.84 \\
7\end{array}$ & 283.8 & 29 & 0.90 \\
\hline
\end{tabular}

Le tableau 2 ci-dessus montre que la valeur de l'eta-carré $\left(\eta^{2}\right)$ est (0.90) pour la compétence de compréhension orale. Cette valeur est supérieure à 0.14 . Cela signifie que la plateforme numérique proposée a un effet significatif $\left(\eta^{2}>0.14\right)$ sur le développement de la compréhension orale en français pour l'échantillon de la recherche. Donc, notre deuxième hypothèse est aussi confirmée. En somme, nous pouvons dire que la plateforme numérique proposée a pu atteindre les objectifs désirés du développement de la compétence de compréhension orale pour notre échantillon de la recherche.

Les résultats de notre recherche sont concordants avec les résultats des études et des recherches antérieures qui affirment que le niveau des étudiants égyptiens des facultés de pédagogie en français langue étrangère est faible surtout à l'oral ( $\mathrm{Al} \mathrm{Adl,} 2017$; El-Alawy, 2015 ; Omar, 2014 ; Mohammed, 2012 ; Mohamed, 2011 ; El-Fakharany, 2010; Hafez, 2009). De même, notre recherche confirme le potentiel pédagogique et éducatif des plateformes numériques pour l'enseignement/apprentissage d'une langue étrangère.

\footnotetext{
* Test T pour échantillons appariés

${ }^{\dagger}$ Carré du test $\mathrm{T}$

†Degré de liberté

${ }^{\S}$ Eta-carré
} 
Plateforme numérique d'apprentissage pour développer les compétences de compréhension orale en FLE

\section{Bibliographie}

Al Adl, W. (2017). Programme d'ordinateur pour développer les compétences de la communication orale chez les étudiants de la section de français aux facultés de Pédagogie (Thèse de doctorat inédite). Université de Damiette, Égypte.

Alrabadi, E. (2011). Quelle méthodologie faut-il adopter pour l'enseignement/apprentissage de l'oral ? Didáctica. Lengua y Literatura, 23, 15-34.

Conseil de l'Europe (2001). Cadre européen commun de référence pour les langues: apprendre, enseigner, évaluer. Paris, France: Les Éditions Didier.

Dankova, N. (2013). Logiciel d'annotation de vidéos FSL : Outil d'aide à la compréhension orale des documents authentiques. Cahiers de l'Ilob, 5, 161-171.

El-Alawy, N. (2015). Utilisation d'une stratégie actionnelle proposée pour développer les compétences de la communication orale des futurs enseignants de FLE à la faculté de pédagogie (Thèse de magistère inédite). Université de Damiette, Égypte.

El-Fakharany, Ch. (2010). Efficacité d'un programme basé sur l'emploi du laboratoire de langues en vue de développer la compétence de la compréhension auditive et son effet sur la conversation en français chez les étudiants de la faculté de pédagogie (Thèse de magistère inédite). Université de Tanta, Égypte. 
Plateforme numérique d'apprentissage pour développer les compétences de compréhension orale en FLE Dr . Walid Sobhi AL ADL

Elkhafaifi, H. (2005). The effect of prelistening activities on listening comprehension in Arabic learners. Foreign Language Annals, 38 (4), 505-513.

Ferroukhi, K. (2009). La compréhension orale et les stratégies d'écoute des élèves apprenant le français en $2^{\text {ème }}$ année moyenne en Algérie. Synergies Algérie, (4), 273-280.

George, S. et Derycke, A. (2005). Conceptions et usages des plates-formes de formation. Revue STICEF, 12, 51-64.

Hafez, H. (2009). Évaluation du traitement didactique de la communication orale en français langue étrangère chez les futurs-enseignants dans les facultés de pédagogie à la lueur de l'enseignement stratégique. Revue de curricula et de méthodologie, Faculté de pédagogie, Université d'Ain-Chams, 152, 1-39.

Huver, E. et Springer, C. (2011). L'évaluation en langues-Nouveaux enjeux et perspectives. Paris, France : Les Éditions Didier.

Hyslop, N. B., \& Tone, B. (1989). Listening: Are we teaching it, and if so, how? Business Communication Quarterly, 52(2), 45-46.

Leroy, D. C. (2016). La compréhension orale en français langue étrangère (Mémoire de maîtrise). Université de Gent, Belgique.

Mohamed, R. (2011). Les croyances des enseignants et des apprenants adultes quant à la rétroaction corrective à l'oral et la pratique réelle en classe de français langue étrangère en Égypte (Thèse de doctorat inédite). Université de Montréal, Canada.

Mohammed, R. (2012, janvier). Les activités ludiques et le développement des compétences communicatives à l'oral en français langue 
Plateforme numérique d'apprentissage pour développer les compétences de compréhension orale en FLE

étrangère (FLE). Revue de la faculté de l'éducation de Kéna,

Université du Sud de la Vallée, Égypte, (15), 1-13.

Mohammed, S. (2007). Efficacité d'un programme basé sur les activités langagières sur le développement de la compréhension et de l'expression orales en français deuxième langue étrangère chez les étudiants du cycle secondaire d'Al Azhar (Thèse de magistère inédite). Université d'Al-Azhar, Égypte.

Omar, H. (2014). Efficacité d'un programme proposé en utilisant le elearning sur le développement des compétences de la communication orale en français chez les étudiants de la section de français, facultés de pédagogie (Thèse de doctorat inédite). Université d'Al-Azhar, Égypte.

Roussel, S. et Tricot, A. (2015). Effet de l'élaboration d'hypothèses sur la compréhension de l'oral et sur les stratégies d'autorégulation de l'écoute en langue seconde : une étude empirique. Apprentissage des Langues et Systèmes d'Information et de Communication (ALSIC), 18, 2-19.

Roussel, S., Rieussec, A., Nespoulous, J. L. et Tricot, A. (2008). Des baladeurs MP3 en classe d'allemand-L'effet de l'autorégulation matérielle de l'écoute sur la compréhension auditive en langue seconde. Apprentissage des Langues et Systèmes d'Information et de Communication (ALSIC), 11(2), 7-37.

Salem, A. (2002 Janvier). L'efficacité de l'enseignement assisté par ordinateur sur le développement de la compétence de communication orale du français chez les étudiants admis aux 
Plateforme numérique d'apprentissage pour développer les compétences de compréhension orale en FLE Dr . Walid Sobhi AL ADL

facultés de pédagogie. Revue de la faculté de pédagogie à Banha, 12(47), 3-55.

Spiezia, R. (2001). Le professeur et l'ordinateur : une approche intégrée de l'enseignement/apprentissage du français dans une université italienne (Thèse de doctorat inédite). Université de Provence, France.

Vandergrift, L. \& Goh, Ch. (2012). Teaching and learning second language listening: Metacognition in action. New York: Routledge. 
Plateforme numérique d'apprentissage pour développer les compétences de compréhension orale en FLE Dr . Walid Sobhi AL ADL

Annexe 1 : Notes des étudiants aux tests de compréhension orale

\begin{tabular}{||c|c|c|c|c||c|c|c|c|c||}
\hline \multirow{2}{*}{$\mathrm{N}^{*}$} & \multicolumn{2}{|c|}{ Pré-CO $^{\dagger}$} & \multicolumn{2}{|c|}{ Pst-CO $^{\dagger}$} & \multicolumn{2}{|c|}{ Pré-CO } & \multicolumn{2}{|c|}{ Pst-CO } \\
\cline { 8 - 11 } & Note & $\%$ & Note & $\%$ & & Note & $\%$ & Note & $\%$ \\
\hline \hline 1 & 10 & 40 & 22 & 88 & 16 & 9 & 36 & 15 & 60 \\
\hline 2 & 10 & 40 & 16 & 64 & 17 & 8 & 32 & 14 & 56 \\
\hline 3 & 7 & 28 & 17 & 68 & 18 & 11 & 44 & 17 & 68 \\
\hline 4 & 10 & 40 & 17 & 68 & 19 & 13 & 52 & 16 & 64 \\
\hline 5 & 6 & 24 & 21 & 84 & 20 & 9 & 36 & 13 & 52 \\
\hline 6 & 6 & 24 & 13 & 52 & 21 & 11 & 44 & 21 & 84 \\
\hline 7 & 9 & 36 & 18 & 72 & 22 & 8 & 32 & 14 & 56 \\
\hline 8 & 9 & 36 & 15 & 60 & 23 & 8 & 32 & 13 & 52 \\
\hline 9 & 5 & 20 & 14 & 56 & 24 & 7 & 28 & 14 & 56 \\
\hline 10 & 10 & 40 & 16 & 64 & 25 & 6 & 24 & 13 & 52 \\
\hline 11 & 8 & 32 & 15 & 60 & 26 & 6 & 24 & 14 & 56 \\
\hline 12 & 4 & 16 & 13 & 52 & 27 & 10 & 40 & 18 & 72 \\
\hline 13 & 11 & 44 & 17 & 68 & 28 & 8 & 32 & 15 & 60 \\
\hline 14 & 5 & 20 & 13 & 52 & 29 & 10 & 40 & 15 & 60 \\
\hline 15 & 9 & 36 & 15 & 60 & 30 & 6 & 24 & 13 & 52 \\
\hline \hline $\mathrm{TN}^{*}$ & 249 & 33 & 467 & 62 & & & & & \\
\hline $\mathrm{M}^{* *}$ & 8.30 & 33 & 15.57 & 62 & & & & & \\
\hline
\end{tabular}

* Numéro de l'étudiant

† Notes à la pré-compréhension orale

Notes à la post-compréhension orale

* Total des notes

** Moyenne arithmétique 